\title{
ON SPRAYS AND SPRAYING IN THE TREATMENT OF CERTAIN SURFACE AFFECTIONS OF THE SKIN.
}

\section{By W. ALLAN JAMIESON, M.D., LL.D., F.R.C.P.E.,* Knight of Grace of the Order of St. John of Jerusalem.}

THe spray, which has firmly established itself as a remedy in morbid states of the larynx and nasopharynx, has been rather neglected when employed as a means of treatment in some affections of the skin. No doubt its usefulness is a restricted one, but it is of great value when suitably employed. Spirituous and oily media are available in its use. It has two great advantages, one of economy and another that it can be directed exactly on the part where it is required. But in the nature of things its action is superficial, and it is only in cases where the epidermic layers alone are disturbed that it can be employed with efficiency.

Its main field of employment is constituted by the hairy parts, which have hitherto been submitted to treatment by sprinkling on lotions or rubbing in soft ointments.

It is therefore in the slighter varieties of seborrhceic dermatitis or in the variety of disturbance of the surface of the scalp known as dandruff, in which showers of white particles are dislodged in combing or brushing the hair, that the spray finds its greatest use.

The spray itself is the ordinary hand one, provided with indiarubber bulb and atomiser. If possible it is best used by another person, who can see exactly where the cloud should be directed. Then it does not matter much how the bulb is placed in relation to the rest of the apparatus, but if the patient must use it for him or herself the bulb is most conveniently placed at the side and not behind.

Two combinations may require to be used, a spirituous and an oily. The spirituous is that of wider applicability. The mixture which I have most largely made use of is one modified from a prescription by Dr. Charles J. White [Journ. Amer. Med. Assoc., 1910, No. 13].

It consists of euresol 2 drms., spiritus formicarum 1 oz., spiritus rosmarini $\frac{1}{2}$ oz., olei ricini $\frac{1}{2} \mathrm{oz}$., spiritus vini rectificati to $8 \mathrm{oz}$.

* This paper was received shortly before Dr. Jamieson's death, and is probably his last contribution to Dermatology. 
Euresol is not at present available, as it is prepared apparently exclusively in Germany. It is an acetate of resorcin, and is met with as a syrupy liquid. For it, therefore, resorcin must be substituted, in the proportion of $1 \mathrm{drm}$. as against 2 of euresol. The main claim made for euresol as against resorcin is that it does not stain white hair, but this claim cannot be fully substantiated.

Should the scalp be reddened and its mobility interfered with, it may be necessary as a preliminary measure to reduce the inflammation and restore pliability by washing with superfatted fluid soap and using sulphur and salicylic pomades. When this has been accomplished, the period for the spray is reached. In case of dandruff or scurf, the spray may be commenced with at once. The mode of procedure is as follows : The scalp must be shampooed once a week or ten days, using for the purpose a mixture of fluid extract of quillaya in warm water, proportion varying from one teaspoonful to one dessertspoonful in half a washhand-basinful of warm water. This is poured on by means of a moderately large sponge and gently rubbed in. The scalp is then dried by means of a warm soft bath-towel. The spray is then used once or twice daily, twice at first, subsequently once. In this way the dandruff rapidly disappears. If the hair has been tending to fall out, the loss speedily diminishes, and is soon arrested more or less completely.

Should the scalp have become atrophied, no new hairs can be expected to reappear. But if it still retains its normal softness, new hairs will reappear.

While this constitutes the wider applicability of the spray, it may also be used as a lubricant in cases where the hair is too dry. When so employed the best medium available is oil of sesame, which has a high reputation among the natives of India as a hair-dressing. Combined with $10 \mathrm{gr}$. of resorcin to the ounce this oil may be sprayed on the scalp once every day, or less often as found necessary. The same treatment may be employed in the case of the beard when this region, as sometimes occurs, becomes affected by dandruff. On no account must the face be washed with soap, plain tepid water being used freely.

Other uses of the spray may be discovered, but the above are those which have proved most efficacious in my hands. 